\title{
Population genetic structure and colonisation of the western Antarctic Peninsula by the seabird tick Ixodes uriae
}

\author{
K. D. McCoy ${ }^{1, *}$, P. Beis ${ }^{1}$, A. Barbosa ${ }^{2,7}$, J. J. Cuervo ${ }^{2,7}$, W. R. Fraser ${ }^{3}$, \\ J. González-Solís ${ }^{4}$, E. Jourdain ${ }^{5,8}$, M. Poisbleau ${ }^{6}$, P. Quillfeldt ${ }^{6}$, E. Léger ${ }^{1}$, M. Dietrich ${ }^{1}$ \\ ${ }^{1}$ Maladies Infectieuses et Vecteurs: Ecologie, Génétique, Evolution et Contrôle, UMR UM1 UM2 CNRS 5290-UR IRD 224, \\ Centre IRD, 34394 Montpellier, France \\ ${ }^{2}$ Dept. Ecología Funcional y Evolutiva, Estación Experimental de Zonas Áridas, CSIC, 04120 Almería, Spain \\ ${ }^{3}$ Polar Oceans Research Group, Sheridan, Montana 59749, USA \\ ${ }^{4}$ Institut de Recerca de la Biodiversitat (IRBio) and Dept. Biologia Animal, Universitat de Barcelona, Barcelona 08028, Spain \\ ${ }^{5}$ Zoonotic Ecology and Epidemiology, Linnaeus University Kalmar, SE-391 82 Kalmar, Sweden \\ ${ }^{6}$ Max Planck Institute for Ornithology, Vogelwarte Radolfzell, 78315 Radolfzell, Germany \\ ${ }^{7}$ Present address: Dept. Ecología Evolutiva, Museo Nacional de Ciencias Naturales, CSIC, 28006 Madrid, Spain \\ ${ }^{8}$ Present address: INRA, UR346, 63122 Saint Genès Champanelle, France
}

\begin{abstract}
Recent observations on the western Antarctic Peninsula have suggested that changing climatic conditions may be increasing pressure on breeding seabirds due to higher exploitation rates by the tick Ixodes uriae. Using data from 8 microsatellite markers and ticks from 6 Pygoscelis spp. colonies, we employed a population genetics approach to specifically test the hypothesis that $I$. uriae is expanding south-westward along the peninsula from the Subantarctic region. Contrary to expectations, tick genetic diversity was high within all colonies, and no remaining signal of colonisation events was evident. Although significant geographic genetic structure occurred among ticks from different colonies, these ectoparasites tended to belong to 2 major genetic groups, one found principally in south-western locations (Palmer Station area) and the other in more north-eastern areas (South Shetland Islands). More central colonies showed a mixture of ticks from each genetic group, suggesting that this area represents a hybridisation zone of ticks from 2 distinct origins. A subsequent clustering analysis, including ticks from 2 Subantarctic locations, did not reveal the source population for the northern peninsula group. Overall, our data refute the hypothesis of a recent south-westward expansion of $I$. uriae along the peninsula and suggest that this tick has been present at more southern latitudes for an extended period of time. Further studies on the distribution and genetic characteristics of this ectoparasite around Antarctica are now required to better understand the colonisation process and predict how changing environmental conditions may affect its presence and diversity in seabird colonies.
\end{abstract}

KEY WORDS: Climate change $\cdot$ Host-parasite interactions $\cdot$ Invasion $\cdot$ Pygoscelis $\cdot$ Seabird population dynamics

\section{INTRODUCTION}

In the region of the Antarctic Peninsula, temperatures have increased substantially over the past $50 \mathrm{yr}$ (Steig et al. 2009), and current research suggests that this trend will continue (Convey et al. 2009). As in other parts of the world, such changes can lead to the redistribution of floral and faunal species and may alter the nature of local interspecific interactions. In particular, increasing temperatures can facilitate the invasion of novel parasitic species, or variants, with potentially significant consequences for endemic 
populations, particularly in combination with other environmental stresses (e.g. Davidson et al. 2011, Wardle et al. 2011). One such organism that may interact with a major component of Antarctic terrestrial fauna is the seabird tick Ixodes uriae White, 1852 (Acari: Ixodidae). This tick is distributed across the circumpolar regions of both hemispheres, exploiting a wide diversity of colonial seabird species (Dietrich et al. 2011). Although abundant in colonies of the Subantarctic (e.g. Bergström et al. 1999a, Frenot et al. 2001), its status in Antarctica is poorly understood (Barbosa \& Palacios 2009). Recent changes in the distribution of seabird species due to sea-ice loss and modifications in prey availability (e.g. Fraser \& Hofmann 2003, Lynch et al. 2008, Trivelpiece et al. 2011), along with changes in climatic conditions have presumably had correlated effects on the distribution and abundance of this parasite.

In colonies where Ixodes uriae is well-established, its numbers can be high and the direct impact on the seabird host strong, with heavy infestations resulting in reduced body condition (Morbey 1996), lower breeding performance (Mangin et al. 2003), nest abandonment (Lynch et al. 2010) and, in some cases, death (Gauthier-Clerc et al. 1998, Bergström et al. 1999b). These changes can lead to modifications in other demographic processes such as site fidelity and recruitment rates, thereby substantially altering seabird population dynamics (Boulinier \& Danchin 1996). In addition to the direct effects of this tick, it also vectors numerous pathogenic organisms (Dietrich et al. 2011). For most of these organisms, we know little to nothing about their pathogenicity for birds, but some can cause human disease (e.g. Borrelia burgdorferi s.l., the Lyme disease agent; Olsén et al. 1993). This suggests that seabirds could play an important role as disease reservoirs and dispersal agents (Gómez-Díaz et al. 2011). The strong spatial and temporal structure of $I$. uriae populations, the range of potential seabird species exploited and the complex interaction between the tick and its vertebrate host may have favoured the evolution of host specialisation in this system (Magalhaes et al. 2007). Indeed, several studies have now demonstrated that genetically distinct populations of this tick exploit different sympatric seabird species (McCoy et al. $2001,2005 b)$ and that the presence of these hostassociated races can have an important impact on tick dispersal (McCoy et al. 2003) and pathogen transmission (Gómez-Díaz et al. 2010). Like most ectoparasites, $I$. uriae shows only weak independent dispersal abilities and must rely on seabird movements for intercolony dispersal (Danchin 1992,
McCoy et al. 2003). In this way, the colonisation process of this parasite depends directly on the behaviour of its specific seabird host.

In a recent study, Barbosa et al. (2011) described the distribution of Ixodes uriae along the western coast of the Antarctic Peninsula and southern Shetland Islands from surveys carried out between 2007 and 2009. While tick prevalence was relatively high in northern areas (8 to $26 \%$ of stones within colonies), no ticks were found in more south-western areas of the peninsula (Yalour Island $65^{\circ} 15^{\prime} \mathrm{S}$, $64^{\circ} 11^{\prime} \mathrm{W}$ and Avian Island $67^{\circ} 46^{\prime} \mathrm{S}, 68^{\circ} 43^{\prime} \mathrm{W}$; see Fig. 1a). Ticks were previously recorded in the Palmer Archipelago as early as the 1980s (Lee \& Baust 1982), and more recent observations suggest the appearance of this tick as far south as Petermann Island $\left(65^{\circ} 10^{\prime} \mathrm{S}, 64^{\circ} 10^{\prime} \mathrm{W}\right)$, where Lynch et al. (2010) noted the first infestations on Adélie penguins in 2006 after more than a decade of working on the island. These authors suggest that increasing temperatures along the peninsula may favour the presence and expansion of this tick to higher latitudes to the detriment of its seabird host. This hypothesis is coherent with a study by Benoit et al. (2009) which showed that tick engorgement rates in the Palmer area were significantly higher during recent prolonged periods of high temperature.

In the present study, we tested the hypothesis that Ixodes uriae populations along the western Antarctic Peninsula have been expanding south-westward from a northern origin. To do this, we employed a population genetic approach based on 8 microsatellite markers. If populations at higher latitudes are truly more recent, we expected lower genetic diversity in these areas, due to colonisation effects (i.e. sequential population bottlenecks), and a significant pattern of genetic isolation with geographic distance from the northeast to the southwest. We also expected ticks in northern areas of the peninsula to be more closely related to ticks from Subantarctic regions. Due to the existence of distinct host races in this tick species, we explicitly took the host factor into account in our analyses.

\section{MATERIALS AND METHODS}

\section{Biological system and sampling sites}

Ixodes uriae is a nidicolous (i.e. nest-inhabiting) tick that is only associated with its seabird host for the bloodmeal which it takes once in each of its 3 life stages (larva-nymph-adult; adult males do not feed) 

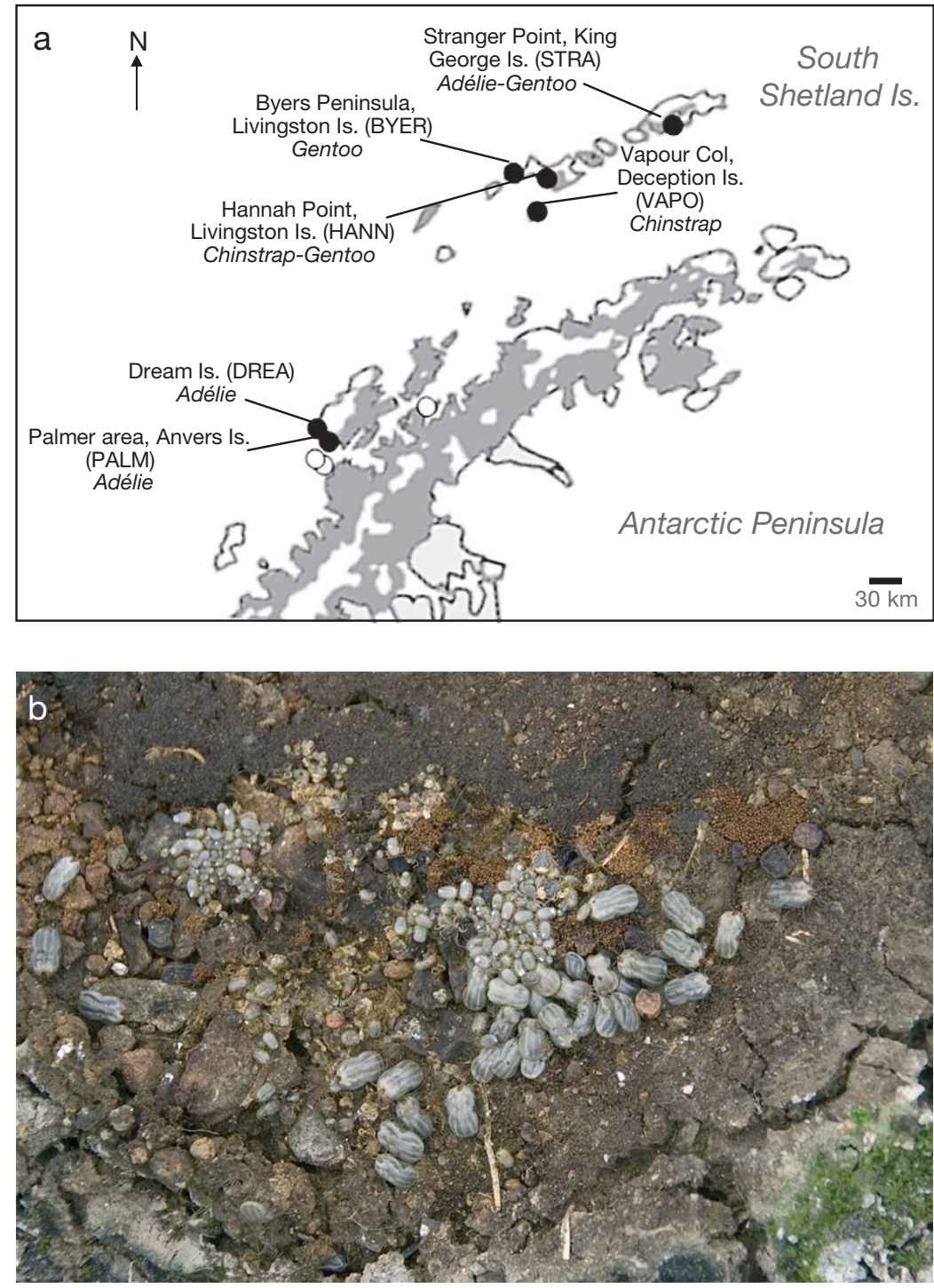

Fig. 1. Ixodes uriae. (a) Known distribution of $I$. uriae along the western coast of the Antarctic Peninsula. Sampled colonies from the present study are represented by black circles; sites where ticks were previously described are indicated by white circles. Host species present in each location are indicated below the colony names. Although chinstrap penguins were present in the HANN colony, the sampled ticks did not exploit these hosts (see 'Results'). Along the peninsula, white areas represent ice sheets; dark grey, ice-free rock; and light grey, areas of ice shelves (modified image from the Landsat Image Mosaic of Antarctica http://lima.usgs.gov/download.php). (b) Different life stages of $I$. uriae within its natural habitat in the Byers Peninsula colony (BYER). Photo: Jacob González-Solís

for a period of between 3 and $12 \mathrm{~d}$, the length increasing with each life stage (Dietrich 2011). The remainder of its life is spent in the substrate surrounding the host nesting area, often in aggregates of several 100 individuals (Benoit et al. 2007; Fig. 1b). This tick reproduces sexually, and a female will lay between 200 and 600 eggs in a single bout before dying (Eveleigh \&
Threlfall 1975, Dietrich 2011). In new seabird colonies, tick populations tend to increase with colony size for approximately the first $30 \mathrm{yr}$, after which time the population dynamics seem to stabilise (Danchin 1992). Tick dispersal depends directly on the intercolony movements of the seabird host and may vary in relation to the population dynamics within the colony (positive or negative colony growth rates; Boulinier \& Danchin 1996) and the behaviour of the specific seabird host species of the colony (McCoy et al. 2003).

As mentioned above, Ixodes uriae can exploit a wide variety of colonial seabirds in circumpolar regions (Dietrich et al. 2011). In the present study, we have focused sampling on ticks associated with 3 penguin species of the genus Pygoscelis that overlap in their distribution along the western Antarctic Peninsula: Adélie ( $P$. adeliae), chinstrap ( $P$. antarctica) and gentoo ( $P$. papua) penguins. Adélie penguin colonies lie around Antarctica and extend north as far as the South Sandwich Islands. These penguins have a strong dependence on the presence of pack ice for breeding/feeding (Trivelpiece et al. 1987), and populations are currently declining along the western coast of the Antarctic Peninsula (Lynch et al. 2008, Trivelpiece et al. 2011). The distribution of the chinstrap penguin is much more restricted, with most individuals breeding in colonies of the South Sandwich Islands and the northern half of the Antarctic Peninsula (Williams 1995). Like the Adélie, these penguins are experiencing severe population declines (Trivelpiece et al. 2011). In contrast to these 2 species, the gentoo penguin has a more northern distribution, with large colonies on the Subantarctic islands (Williams 1995); the gentoo penguin is currently undergoing a vast population expansion southward along the peninsula (Lynch et al. 2008). It is during the austral summer (November to March), when these 3 penguin species are reproducing, that the tick takes its bloodmeal. 
Ticks were collected over a $10 \mathrm{yr}$ period (2000 to 2010) from under stones within colonies at the end of the breeding period at 6 locations along the Antarctic Peninsula (Fig. 1, Table 1). All tick stages were collected and conserved in $70 \%$ ethanol until analyses. Two of the 6 colony areas sampled were heterospecific in nature (i.e. containing 2 of the 3 species considered). As host use could not be assured for these ticks, we carried out a posteriori host identifications using DNA remnants contained in the tick gut (see following subsection).

To investigate a possible northern origin for ticks of the peninsula, we also analysed available specimens from 2 locations in the Subantarctic region (Table 1): Magdalena Island (MAGD), Chile ( $44^{\circ} 39^{\prime} \mathrm{S}$, $73^{\circ} 8^{\prime} \mathrm{W}$ ), where ticks were sampled from Magellanic penguins Spheniscus magellanicus, and New Island (NEWI), Falklands $\left(51^{\circ} 43^{\prime} \mathrm{S}, 61^{\circ} 19^{\prime} \mathrm{W}\right)$ where ticks were collected from black-browed albatross (BBA) Diomedea melanophris, rockhopper penguins (RP) Eudyptes chrysocome and imperial shags (IS) Phalacrocorax atriceps. These populations lie, respectively, $\sim 2500$ and $\sim 870 \mathrm{~km}$ northwest of the South Shetland Islands. On NEWI, ticks were collected directly from the birds.

\section{Molecular analyses}

DNA from approximately 30 ticks (adult females and nymphs) was extracted for each sampling lo- cation (and host species, in the case of NEWI), taking care to select ticks from a maximum number of aggregation sites within each colony. DNA extractions were carried out using the DNeasy Blood and Tissue kit (Qiagen) following the manufacturer's protocol. The extracted DNA of each tick was quantified by a spectrophotometric analysis (Nanodrop, Labtech) and was diluted to a final standardised volume of $50 \mathrm{ng}^{-1} \mathrm{l}^{-1}$.

To examine tick genetic diversity and population structure, ticks were genotyped at 8 microsatellite markers previously developed and used for this species (McCoy \& Tirard 2000). DNA amplifications and allele size determination were carried out as previously described in Kempf et al. (2009) and Dietrich et al. (2012).

To determine host use in the mixed Pygoscelis spp. colonies (Stranger Point [STRA], Hannah Point [HANN]), we amplified a portion of the cytochrome $C$ oxidase 1 (CO1) gene using the DNA extractions of individual ticks. Host DNA was amplified using a nested polymerase chain reaction (PCR) method according to the protocol of Tavares \& Baker (2008), except that reactions were performed in a total volume of $25 \mu \mathrm{l}$ rather than $12.5 \mu \mathrm{l}$. The primers for the first amplification were LTyr and COI907Ah2. For the second PCR amplification, $2 \mu \mathrm{l}$ of the amplicon from the first reaction was used as the DNA template along with the internal primers COIaRt and COI748Ht. Host DNA amplification was revealed by the presence of a $700 \mathrm{bp}$ amplification product on a $2 \%$ agarose gel. All amplified products were subse-

Table 1. Ixodes uriae. Sampling details and genetic diversity of I. uriae populations from the north-western area of the Antarctic Peninsula and from 2 potential Subantarctic source areas. Colony codes are the same as in Fig. 1 and are indicated after the sampling location. 'Year' refers to the years when ticks were sampled at each site, and ' $n$ ' to the number of genotyped ticks. $F_{\text {IS }}$ estimates are provided with all microsatellite loci $\left(F_{\mathrm{IS}}\right)$ and without markers that showed consistent patterns of null alleles $\left(F_{\mathrm{IS}}{ }^{*}\right)$ (see 'Results'). $F_{\mathrm{IS}}$ estimates that were significantly different from zero after Bonferroni correction are indicated in bold. Values of genetic diversity $\left(H_{\mathrm{s}}\right)$ and allelic richness $(A R)$ are across-locus averages; $A R$ is based on 16 diploid individuals randomly sampled in each population

\begin{tabular}{|llcccccc}
\hline Sampling location & Host & Year & $\mathrm{n}$ & $F_{\mathrm{IS}}$ & $F_{\mathrm{IS}}{ }^{*}$ & $H_{\mathrm{S}}$ & $A R$ \\
\hline North-western Antarctic Peninsula & & & & & & \\
Stranger Point (STRA) & Adélie penguin, & 2007,2010 & 32 & $\mathbf{0 . 2 1 8}$ & 0.116 & 0.68 & 6.45 \\
& Gentoo penguin & & & & \\
Hannah Point (HANN) & Gentoo penguin & 2009,2010 & 32 & $\mathbf{0 . 1 6 0}$ & 0.109 & 0.70 & 6.57 \\
Byers Peninsula (BYER) & Gentoo penguin & 2009 & 31 & $\mathbf{0 . 2 0 3}$ & 0.114 & 0.66 & 6.17 \\
Vapour Col (VAPO) & Chinstrap penguin & 2010 & 33 & $\mathbf{0 . 1 5 8}$ & 0.085 & 0.67 & 5.67 \\
Dream Island (DREA) & Adélie penguin & 2000,2001 & 18 & $\mathbf{0 . 2 5 4}$ & 0.194 & 0.68 & 6.61 \\
Palmer area (PALM) & Adélie penguin & 2000 & 28 & $\mathbf{0 . 2 1 9}$ & 0.144 & 0.64 & 5.92 \\
Subantarctic areas & & & & & & \\
Magdalena Island (MAGD) & Magellanic penguin & 2009 & 24 & $\mathbf{0 . 0 7 2}$ & -0.890 & 0.65 & 6.68 \\
New Island (NEWI) & Rockhopper penguin & 2009 & 32 & $\mathbf{0 . 2 3 8}$ & 0.111 & 0.71 & 7.87 \\
New Island & Black-browed albatross & 2009 & 30 & $\mathbf{0 . 3 2 7}$ & 0.276 & 0.67 & 7.38 \\
New Island & Imperial shag & 2009 & 31 & $\mathbf{0 . 2 4 3}$ & 0.124 & 0.72 & 8.50 \\
\hline
\end{tabular}


quently sent for direct sequencing (Eurofins MWG Operon). Sequence chromographs were manually verified, and a final fragment of $385 \mathrm{bp}$ was used for host identification. A BLAST sequence analysis (NCBI, National Library of Medicine) was then carried out for each distinct haplotype. We were able to successfully assess host use for $75.3 \%$ of the tested ticks using this method.

\section{Statistical analyses}

Based on a preliminary analysis that indicated no difference in allele frequencies among ticks collected at different points in time (data not shown), we treated all ticks collected at a given sampling location as a single population, irrespective of the sampling year. Preliminary analyses delimited when host species was to be taken into account for defining populations (Table 1).

We first tested for linkage disequilibrium among our 8 loci to verify that they could be considered as independent replicates of the tick genome; using the GENEPOP software V.4.1. (Raymond \& Rousset 1995), a probability test for each pair of loci in each population was computed using the Markov chain algorithm. Given the large number of tests, significance was evaluated using a generalised binomial approach (de Meeûs et al. 2009). Global tests for each locus pair were calculated using Fisher's method. We then tested all loci and populations for deviations from Hardy-Weinberg proportions using FSTAT V.2.9.3 (Goudet 1995). Deviations from Hardy-Weinberg proportions were measured in each population by the estimator $F_{\text {IS }}$ (Weir \& Cockerham 1984) and tested for significance by randomising alleles among individuals within samples (5000 permutations) and correcting for multiple tests (Bonferroni procedure). Heterozygote deficits associated with a particular locus can often be explained by the presence of null alleles (de Meeûs et al. 2007) and may be present in our study as the loci employed were developed on Ixodes uriae samples from the northern hemisphere, which are genetically isolated from those in the south (McCoy et al. 2005b). We therefore tested our markers for the presence of null alleles using the software MICRO-CHECKER V.2.2.3 (Van Oosterhout et al. 2004).

Two estimates of genetic diversity were calculated for each population using FSTAT V.2.9.3 (Goudet 1995): allelic richness, corrected for the number of sampled individuals and Nei's diversity index, $H_{\mathrm{s}}$.
We tested for a significant difference in diversity among colonies using a 1-way analysis of variance (ANOVA; or Kruskal-Wallis test if normality was not met) on standardised diversity values (z-values). To test for a reduction in genetic diversity from northeast to southwest, we tested for a negative correlation between these diversity measures and latitude by calculating Spearman correlation coefficients ( $R$ V.2.12.2).

Population structure along the peninsula was initially examined using the GENEPOP software V.4.1 (Raymond \& Rousset 1995). We measured the degree of differentiation among colonies using Weir \& Cockerham's (1984) estimator of Wright's $F_{\mathrm{ST}}$. Significant deviation from the null hypothesis of no differentiation among colonies was tested using an exact probability test (Goudet et al. 1996). To understand how this differentiation changed with the geographic distance between populations, we tested for patterns of isolation by distance using a Mantel test of the genetic distance [measured as $F_{\mathrm{ST}} /\left(1-F_{\mathrm{ST}}\right)$ ] and the geographic distance (log distance) between each population pair, with 10000 permutations of genotypes among populations (IBDWS software V.3.14: Jensen et al. 2005).

We then examined the number of genetically distinct pools of individuals present within our sampled populations from the peninsula using a Bayesian clustering approach with STRUCTURE V.2.3.1 (Pritchard et al. 2000). We included all tick individuals and set the number of potential clusters $(K)$ from 1 to 10 , with 5 independent runs. Computations were run under the admixture model with correlated allelic frequencies. We used the LOCPRIOR model that takes into account the original population of each tick individual; this model is recommended in cases when the signal of genetic structure is weak (Hubisz et al. 2009). Simulations were carried out using a burn-in of 100000 iterations, allowing for the stabilization of summary statistics, followed by a run length of 100000 iterations. The most probable number of populations was inferred by Evanno's method $(\Delta K)$ (Evanno et al. 2005).

Finally, to test whether we could identify a source population for ticks of the peninsula, we carried out a second STRUCTURE analysis including the genetically distinct populations from the peninsula (see 'Results') and those from the 2 locations in the Subantarctic region. Conditions for running STRUCTURE were as above, except that $K$ varied from 1 to 9 (5 populations on the peninsula, 1 on MAGD, 3 on NEWI corresponding to each host-associated tick population; see 'Results'). 


\section{RESULTS}

Among the 280 tests of linkage disequilibrium (10 populations $\times 28$ locus pairs), only 10 pairs were below the $5 \%$ significance threshold. This is less than the number of significant tests expected by chance alone given the number of tests $\left(k^{\prime}=21\right.$, generalised binomial procedure, MultiTest V.1.2; de Meeûs et al. 2009), and no locus pair showed evidence of linkage when tests were combined across populations (all p-values $>0.28$, Fisher's method); the 8 loci used can therefore be considered as statistically independent. Initially, all populations showed significant deviations from Hardy-Weinberg proportions (Table 1). These deviations were largely explained by heterozygote deficits at 2 loci in the peninsula populations, T3 and T5. Indeed, the presence of null alleles was suggested for these loci by our analysis with MICROCHECKER. When these loci were removed from the analysis, Hardy-Weinberg proportions were found in all 6 populations (Table 1). In Subantarctic populations, deviations could not be explained by the same loci. In these populations, it was T38 and T44 that frequently showed signs of null alleles (Table 1). Although, the presence of null alleles can add noise to our estimates of population structure, the biological significance of the results presented here do not change whether or not these loci are included. For this reason, we have included all 8 loci in the results that follow.

Molecular analyses of host use indicated that the sampled ticks used both host species present in the STRA colony $(78 \%$ gentoo, $22 \%$ Adélie, $\mathrm{n}=18)$. However, only gentoo penguins were exploited in the HANN colony $(\mathrm{n}=27)$, despite the presence of chinstrap penguins. In the monospecific colonies tested as controls for host use, sequences always identified the correct host species; chinstrap penguins for Vapour Col (VAPO, $\mathrm{n}=3$ ), gentoo penguins for Byers Peninsula (BYER, $\mathrm{n}=6$ ) and Adélie penguins for the colonies of Palmer area and Dream Island (PALM and DREA, respectively, $\mathrm{n}=7$ ).

Genetic diversity did not vary significantly among peninsula populations for either Nei's diversity index, $H_{\mathrm{s}}(F=0.208, \mathrm{df}=5$, $\mathrm{p}=0.957)$ or allelic richness $(F=$
0.414, $\mathrm{df}=5, \mathrm{p}=0.836$ ). When the potential source populations are included, there was no difference among colonies in Nei's diversity index $(H=11.23$, $\mathrm{df}=9, \mathrm{p}=0.261$ ), but the NEWI populations tended to show higher allelic richness than the others $(F=3.63$, $\mathrm{df}=9, \mathrm{p}<0.001$ ) (Table 1). There was little indication that diversity declined with increasing latitude along the peninsula (Table 1; Spearman correlation tests for Nei's diversity index $\rho_{H_{\mathrm{s}}}=-0.71, \mathrm{p}=0.07$; for allelic richness $\rho_{A R}=-0.26, p=0.33$ ).

The global structure among tick populations of the peninsula was weak, but significant $\left(F_{\mathrm{ST}}=0.025 \pm\right.$ $0.012, \mathrm{p}<0.0001$ ); all population pairs showed significantly different allele frequencies except those of HANN, DREA and PALM (Table 2). In contrast, peninsula populations were all strongly distinct from the NEWI and MADG populations (Table 2).

There was a significant pattern of isolation by distance among peninsula populations $(\mathrm{r}=0.51, \mathrm{p}=$ $0.0041)$; the probability of tick dispersal is therefore linked to the geographic distance among colonies, irrespective of the host species present. These patterns suggest that there is little association between tick population structure and the different seabird species present in each of the peninsula colonies. For example, tick populations from the PALM and STRA were the most strongly differentiated (Table 2), despite the fact that ticks used Adélie penguins in both areas. The lack of host-associated structure contrasts with previous results in Subantarctic islands (see McCoy et al. 2005b) and with those on NEWI, where significant host-associated structure is clearly apparent (Table 2).

Table 2. Ixodes uriae. Pairwise genetic differentiation between tick populations of the Antarctic Peninsula region, along with potential source populations, as estimated by the $F_{\mathrm{ST}}$ index. Estimates in bold are significantly different from zero after Bonferroni correction ( $\mathrm{p}$-values < 0.001). Population codes as described in Fig. 1 and Table 1. BBA: black-browed albatross; IS: imperial shag; RP: rockhopper penguin

\begin{tabular}{|c|c|c|c|c|c|c|c|c|}
\hline & \multicolumn{4}{|c|}{ Antarctic Peninsula populations } & \multicolumn{4}{|c|}{ Potential source populations } \\
\hline & HANN BYER & VAPO & DREA & PALM & MAGD & $\begin{array}{l}\text { NEWI } \\
\text { (BBA) }\end{array}$ & $\begin{array}{c}\text { NEWI } \\
\text { (IS) }\end{array}$ & $\begin{array}{c}\text { NEWI } \\
\text { (RP) }\end{array}$ \\
\hline \multicolumn{9}{|c|}{ Antarctic Peninsula populations } \\
\hline STRA & 0.01230 .0360 & 0.0347 & 0.0432 & 0.0498 & 0.2880 & 0.2173 & 0.2473 & 0.2215 \\
\hline HANN & 0.0177 & 0.0134 & 0.0111 & 0.0070 & 0.2830 & 0.2104 & 0.2328 & 0.2121 \\
\hline BYER & & 0.0211 & 0.0393 & 0.0204 & 0.2929 & 0.2255 & 0.2481 & 0.2244 \\
\hline VAPO & & & 0.0249 & 0.0241 & 0.2991 & 0.2236 & 0.2424 & 0.2320 \\
\hline DREA & & & & 0.0072 & 0.2920 & 0.2183 & 0.2338 & 0.2157 \\
\hline PALM & & & & & 0.3132 & 0.2448 & 0.2578 & 0.2377 \\
\hline \multicolumn{9}{|c|}{ Potential source populations } \\
\hline MAGD & & & & & & 0.1168 & 0.0736 & 0.0378 \\
\hline NEWI ( & BBA) & & & & & & 0.0764 & 0.0448 \\
\hline NEWI ( & & & & & & & & 0.0405 \\
\hline
\end{tabular}


The analyses in STRUCTURE suggested that the most probable number of populations for the tick individuals sampled along the peninsula was $K=2$. From Fig. 2, one can distinguish 3 population groups made up of differing proportions of ticks from the 2 genetic groups: all individuals from the DREA and PALM populations are assigned to Group 1, whereas individuals from STRA tend to belong to Group 2. The populations that lie in an intermediate position (HANN, BYER, VAPO) show a mix of the 2 genetic groups. We therefore seem to have distinct population groups in the most southern and northern sites, and a mix of these genetic entities in between.
The second STRUCTURE analysis, that included ticks from 2 Subantarctic locations, also found that the most probable number of groups was $K=2$ and divided ticks into one peninsula group and a second Subantarctic group (Fig. 3). Interestingly, if the number of groups is increased to 3, we see the start of host-associated structure on NEWI (Fig. 3), supporting the pairwise $F_{\mathrm{ST}}$ estimates cited above. If $K$ is increased to 4 , we then also see the appearance of the 2 genetic groups on the peninsula (Fig. 3). In no case is there any suggestion of mixing between the ticks of the peninsula and those of the Subantarctic islands considered.

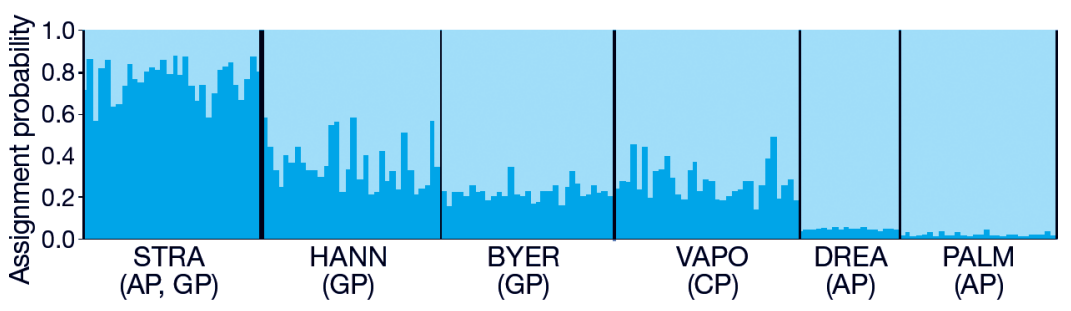

Fig. 2. Ixodes uriae. Tick population structure inferred by Bayesian clustering within the western region of the Antarctic Peninsula. Each tick individual is represented by a thin vertical bar composed of $K$ segments, the length of which is proportional to the probability that the tick belongs to each genetic pool (respectively, dark and light blue, here, $K=2$ ). Colony acronyms are the same as in Fig. 1 and Table 1. Seabird hosts used by ticks in each colony are shown below the colony name. GP: gentoo penguin; CP: chinstrap penguin; AP: Adélie penguin

\section{DISCUSSION}

In the present study, we tested for genetic evidence of a recent southward expansion of the tick Ixodes uriae along the western Antarctic Peninsula, an expansion which could be related to changing environmental conditions and associated changes in seabird population dynamics. Interestingly, the genetic data that we obtained do not support this hypothesis. Indeed, genetic diversity was similar

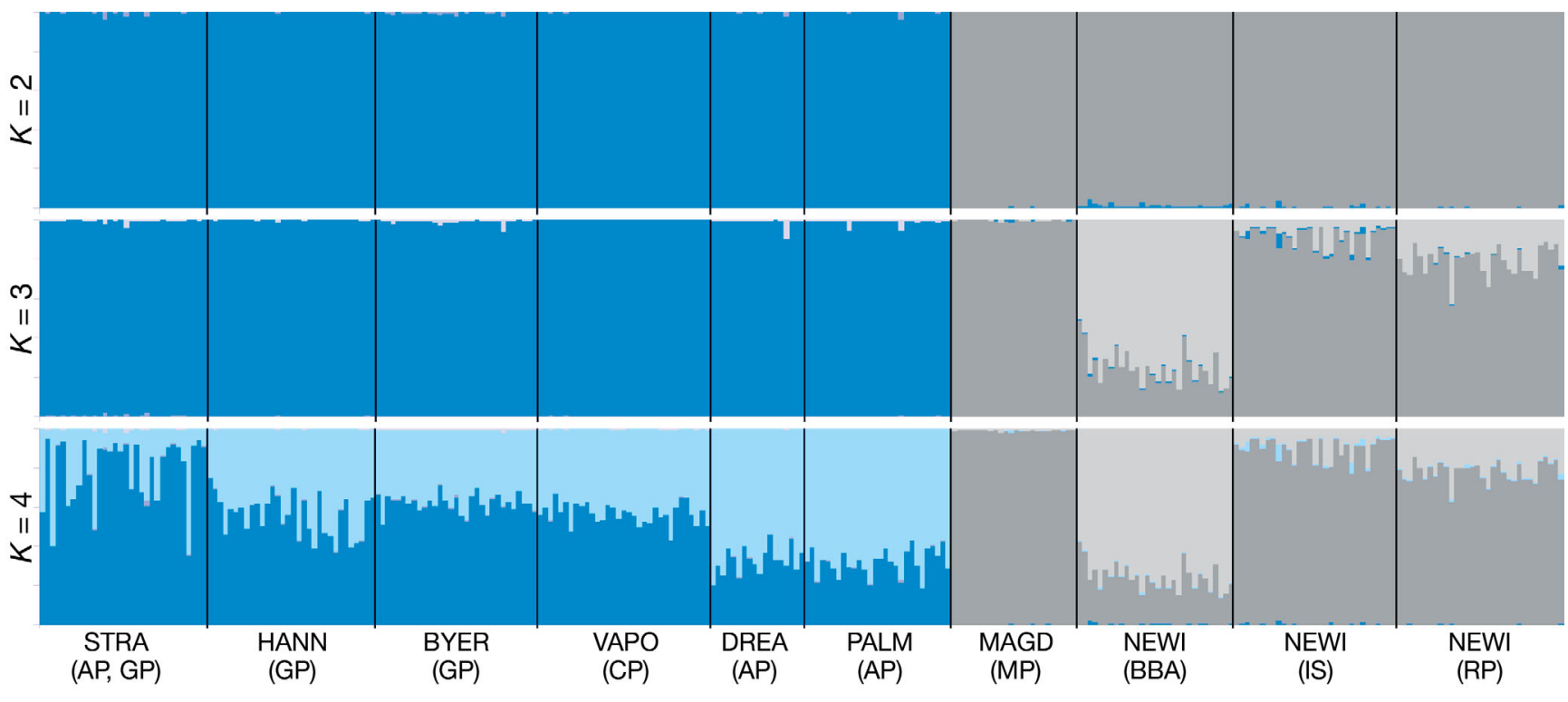

Fig. 3. Ixodes uriae. Population structure among ticks from the western region of the Antarctic Peninsula and 2 Subantarctic colonies (Isla Magdalena, Chile, and New Island, Falklands) inferred by Bayesian clustering. Colony acronyms are the same as in Fig. 1 and Table 1. Each tick individual is represented by a thin vertical bar composed of $K$ segments, the length of which is proportional to the probability that the tick belongs to each genetic pool (here, $K=2$ was selected as the most probable number of groups, blue and grey colours). Increasing $K$ to 3 or 4 did not reveal any mixing between the 2 geographical regions (see 'Results' for more detail). Seabird hosts present in the New Island colony are shown below the colony name. BBA: black-browed albatross; IS: imperial shag; RP: rockhopper penguin; MP: Magellanic penguins; other abbreviations for seabirds as in Fig. 2 
among the peninsula colonies, suggesting that any traces of colonisation bottlenecks have been erased. Although structure among populations was evident, this structure was relatively weak and largely based on the relative distance among colonies. Contrary to our prediction of a recent south-westward colonisation of Pygocelis spp. populations by ticks coming from the north, our results suggest rather that north-eastern and south-western populations along the peninsula originate from 2 distinct source populations. These results are particularly surprising given that previous studies have suggested the quasi absence of $I$. uriae in southern areas of the peninsula (Barbosa et al. 2011) and require that we revisit our understanding of this parasite's distribution and the factors that affect it.

The tick samples we used in the present study were collected over a $10 \mathrm{yr}$ period. Temporal variation in sampling can be a confounding factor when measuring population structure as temporal changes in allele frequencies can occur within colonies (McCoy 2009). However, several aspects argue against this type of variation being responsible for the patterns of structure we found. First, a preliminary analysis of tick samples taken at a $3 \mathrm{yr}$ interval in the STRA colony showed no significant temporal structure. Likewise, tick populations sampled in different years (STRA, HANN, DREA) did not show significant deviations from Hardy-Weinberg proportions after accounting for loci with null alleles; we would have found heterozygote deficits in these locations due to a Wahlund effect if distinct populations had been combined (de Meeûs et al. 2007). Finally, genetic diversity estimates were high and similar across the colonies of the peninsula. As 100s of ticks can often be found under a single stone within colonies (e.g. Benoit et al. 2007, Barbosa et al. 2011; see Fig. 1b), these findings suggest that tick population sizes are large and that most populations likely maintain equilibrium genotype frequencies across generations (i.e. the effect of genetic drift is weak). For these reasons, we believe our results to be robust to sampling artefacts.

The host represents an essential component of a parasite's environment and therefore plays a key role in the evolution of these populations (Combes 2001). Although only on the seabird for a short period during each bloodmeal, the interaction between the tick and its host is intense, involving the production of a complex array of bio-active molecules (e.g. Brossard \& Wikel 2004). Several studies have demonstrated the evolution of host-specific tick races in Ixodes uriae (McCoy et al. 2001, 2005b), and experimental work has shown an adaptive basis for these patterns (Dietrich 2011). We found no evidence of host-related population structure in our data from the western Antarctic Peninsula. Ticks that exploited Adélie penguins were present in the most south-western and the most north-eastern colonies sampled, and were strongly differentiated, whereas ticks sampled from gentoo penguins in the HANN colony were genetically similar to those from Adélie penguins in the PALM and DREA colonies. This contrasts with patterns found in the northern hemisphere (McCoy et al. 2001, 2005b, Kempf et al. 2009) and on NEWI (present study), where sympatrically occurring seabird species tend to have distinct tick populations. However, no genetic structure was found between tick populations exploiting closely related Eudyptes spp. penguins (E. chrysocome and E. chrysolophus) in the Crozet Archipelago, despite significant structure between these ticks and those in parapatric colonies of the king penguin Aptenodytes patagonicus. Dietrich et al. (2012) also found a lack of host-associated structure in ticks exploiting sibling seabird species in the North Pacific. Previous field observations from the peninsula suggested some segregation of ticks among different seabird species; Lynch et al. (2010) noted that ticks were only observed to exploit Adélie penguins on Petermann Island, despite the presence of gentoo penguins in the same colony. It is therefore possible that the genetic patterns we see are due to a recent host shift of ticks that were primarily adapted to Adélie penguins in the south, to chinstrap and gentoo penguins in more northern locations. Adaptation to these newly colonised host species may then follow, and genetic differentiation may build up over time. Alternatively, it may be that the host species we consider in the present study are phylogenetically and/or ecologically similar enough to represent analogous environments for ticks (McCoy et al. 2005b, Dietrich et al. 2012) and the patterns seen on Petermann Island reflected some form of temporary host segregation. Experimental studies to examine the specific constraints that may act in tick specialisation and a broader host sampling will be required to help understand whether the patterns we see are due to a host shift or a lack of host specialisation.

We found the greatest genetic differentiation in Ixodes uriae populations between the STRA colony (in the northeast) and DREA/PALM colonies (in the southwest). The STRUCTURE analysis identified these ticks as coming from 2 distinct genetic pools, with the 3 colonies in intermediate geographic positions (HANN, BYER, VAPO) containing a mix of ticks from these 2 genetic sources. At the northern end of the peninsula, there are several possible source populations for I. uriae, with large seabird populations 
present at the tip of South America and on South Georgia and the South Sandwich Islands. Although we were not able to sample ticks from any Pygoscelis spp. in South America, tick samples from other species were not suggestive of a South American source for the ticks from the Antarctic Peninsula. Indeed, even among-locus patterns of amplification differed between ticks from the Subantarctic colonies of South America and those of the Antarctic Peninsula (see results on the presence of null alleles), suggesting significant isolation among these tick groups. The large Pygoscelis spp. colonies of South Georgia and the South Sandwich Islands were not sampled in the present study, but may be likely candidates for the origin and maintenance of this northern group.

The situation at the southern end of the peninsula is more complex. Only 1 tick population has been officially recorded further south than PALM, from Petermann Island (Lynch et al. 2010), and the infestation status of other seabird colonies in this region and along the Antarctic continent are largely unknown. There has been 1 record of ticks from Ross Island associated with the southern skua Catharacta antarctica (Wilson 1967) and periodic field observations of ticks on Avian Island (W. R. Fraser pers. obs.). It may be that ticks are present at these more southern latitudes, but due to harsh environmental conditions have been maintained at very low population sizes. Indeed, the occupation history of this area by Adélie penguins has changed substantially over time in relation to climate, with major alternations in population sizes between glacial and warming periods (Emslie \& McDaniel 2002), and recent changes in response to ice sheet cover and prey availability (e.g. Croxall et al. 2002, Fraser \& Hofmann 2003, Forcada et al. 2006, Lynch et al. 2008, Trivelpiece et al. 2011). Tick populations may therefore have been present in this area for 1000s of years and only now, with warming temperatures and associated changes in precipitation, are increasing in abundance to the point where they may impact seabird populations. Rigorous sampling efforts in colonies from around the Antarctic continent and from a variety of potential host species will thus be required to understand the precise source of these 2 genetic pools of ticks and whether increasing tick infestations may join other environmental stresses (Le Bohec et al. 2008) to exacerbate declines in penguin populations.

Among-colony dispersal of ticks is, by definition, linked to the movements and behaviour of the seabird host (e.g. McCoy et al. 2003). Although information is still scarce, penguin populations do not seem to be strongly genetically structured (Roeder et al. 2001, Dranitsina et al. 2006, Bouzat et al. 2009). For example, Roeder et al. (2001) found no evidence of population structure in Adélie penguins from around the Antarctic continent. This lack of structure could be due in part to the large population sizes of these species, but also suggests that some intercolony movement occurs. Indeed, site fidelity in Pygoscelis spp. penguins may be lower than previously thought, particularly under conditions of stress (Dugger et al. 2010). As dispersal in Ixodes uriae is thought to occur primarily when failed breeders or young birds prospect for new nest sites/colonies (Danchin 1992), the structure we find in tick populations can reveal some essential information about the population functioning of their penguin hosts. For example, the fact that south-western tick populations on Adélies (PALM/DREA) are genetically distinct from those on King George Island (STRA) suggests little movement of these birds northward. There is currently a $400 \mathrm{~km}$ gap in the distribution of Adélie breeding colonies along the western Antarctic Peninsula, from the area around Palmer Station to Nelson Island in the South Shetlands (Woehler 1993). A study of stable isotopes in eggshells has shown that this 'Adélie gap' is also maintained in winter (Polito et al. 2011) and therefore likely represents an important barrier to tick dispersal. In contrast, among-colony movements along the peninsula in the other 2 Pygoscelis species considered here appear more likely and may explain some of the mixed ancestry of ticks that we see in the HANN, BYER and VAPO colonies. For example, although gentoo penguins tend to remain close to the breeding colony in winter (Williams 1995), their populations are currently expanding southward along the peninsula, and birds may carry ticks between colonies while prospecting for breeding sites. Chinstrap penguins migrate considerably greater distances from the breeding colony than gentoos, moving northward after reproduction for the moult (Williams 1995, Biuw et al. 2010). Such movements could be responsible for some tick dispersal, but will depend on how long ticks can remain attached to the host during this relatively long migration (Biuw et al. 2010). Depending on patterns of host specificity (see above), other species that frequent Pygoscelis spp. colonies may also be responsible for tick dispersal at different spatial scales (e.g. predators such as skuas Catharacta spp. or giant petrels Macronectes giganteus). However, for now, we have almost no information on the use of these alternative hosts by I. uriae. More data on patterns of host specificity and on the movements of individual birds are thus required to better understand intercolony dispersal in this tick. 


\section{CONCLUSIONS}

Our results did not support our initial hypothesis of a recent and northern origin for tick Ixodes uriae populations along the Antarctic Peninsula. However, these analyses revealed an apparently complex functioning of tick populations in this region of the world, with ticks from 2 distinct genetic sources mixing along the peninsula. Future work will require a more complete sampling of ticks from around the Antarctic and Subantarctic regions and from a greater range of host species. As reproductive potential is higher in this tick compared to in its seabird host, population structure may evolve more rapidly in the parasite. Given the complete dependence of this tick on its host for intercolony dispersal, understanding patterns of population structure and functioning in the parasite can provide complementary insights into the ecology and demography of seabirds (McCoy et al. 2005a) and the dissemination risk for other parasites and pathogens. As I. uriae is present in high latitudes of both hemispheres, systematic monitoring (for example, in relation to seabird population monitoring) would be advisable to better comprehend the factors that affect the population dynamics of this ectoparasite, the possible consequence of these dynamics for seabird populations, and the role of a changing environment on these patterns.

Acknowledgements. We thank V. Noel, C. Chevillon, Y. Michalakis, B. Olsen, E. de Mas, J. Hernandez, D. González Acuña, L. Demongin, A. Quesada, E. Elguero and T. Boulinier for assistance at various stages of this work. Data used in this study were partly produced using the technical facilities of the IFR119 'Montpellier Environnement Biodiversité'. Financial support was provided by the European Regional Development Fund, the Ministerio de Ciencia e Innovacion from the Spanish Government (Projects POL2006-06635, POL2006-05175，CGL2004-01348，CGL2007-60369， CGL 2009-11278/BOS, CMT 2011-24427) and the Agence National de la Recherche, France (ANR-06-JCJC-0095-01). Fieldwork on New Island was made possible by support from the New Island Conservation Trust and financial support from the Deutsche Forschungsgemeinschaft (DFG, Qu 148-1 ff.). M.D. was supported by a fellowship from the French Ministry for National Education and Research, and E.L., by a PhD scholarship from the University of Montpellier I, France. Permission for tick collection was granted by the Spanish Polar Committee. We thank the Spanish Antarctic base 'Gabriel de Castilla', the Argentinean Antarctic base 'Teniente Jubany', the Spanish Polar ship 'Las Palmas', the Maritime Logistic Unit (CSIC) and the Chilean Antarctic Secretariat and the Argentinean Antarctic Institute for logistic support and transport. This study is a contribution to the International Polar Year Project 172 BIRDHEALTH and to the PINGUCLIM project.

\section{LITERATURE CITED}

Barbosa A, Palacios MJ (2009) Health of Antarctic birds: a review of their parasites, pathogens and diseases. Polar Biol 32:1095-1115

Barbosa A, Benzal J, Vidal V, D'Amico V and others (2011) Seabird tick (Ixodes uriae) distribution along the Antarctic Peninsula. Polar Biol 34:1621-1624

Benoit JB, Yoder JA, Lopez-Martinez G, Elnitsky MA, Lee RE, Denlinger DL (2007) Habitat requirements of the seabird tick, Ixodes uriae (Acari: Ixodidae), from the Antarctic Peninsula in relation to water balance characteristics of eggs, nonfed and engorged stages. J Comp Physiol B 177:205-215

> Benoit JB, Lopez-Martinez G, Elnitsky MA, Lee RE, Denlinger DL (2009) Increase in feeding by the tick, Ixodes uriae, on Adélie penguins during a prolonged summer. Antarct Sci 21:151-152

Bergström S, Haemig PD, Olsen B (1999a) Distribution and abundance of the tick Ixodes uriae in a diverse subantarctic seabird community. J Parasitol 85:25-27

Bergström S, Haemig PD, Olsen B (1999b) Increased mortality of black-browed albatross chicks at a colony heavily infested with the tick Ixodes uriae. Int J Parasitol 29: 1359-1361

Biuw M, Lydersen C, De Bruyn PJN, Arriola A, Hofmeyr GGJ, Kritzinger P, Kovacs KM (2010) Long-range migration of a chinstrap penguin from Bouvetøya to Montagu Island, South Sandwich Islands. Antarct Sci 22:157-162

> Boulinier T, Danchin E (1996) Population trends in kittiwake Rissa tridactyla colonies in relation to tick infestation. Ibis 138:326-334

> Bouzat JL, Walker BG, Boersma PD (2009) Regional genetic structure in the Magellanic penguin (Spheniscus magellanicus) suggests metapopulation dynamics. Auk 126: 326-334

> Brossard M, Wikel SK (2004) Tick immunobiology. Parasitology 129:161-176

Combes C (2001) Parasitism: the ecology and evolution of intimate interactions. University of Chicago Press, Chicago, IL

- Convey P, Bindschadler R, Di Prisco G, Fahrbach E and others (2009) Antarctic climate change and the environment. Antarct Sci 21:541-563

Croxall JP, Trathan PN, Murphy EJ (2002) Environmental change and Antarctic seabird populations. Science 297: 1510-1514

> Danchin E (1992) The incidence of the tick parasite Ixodes uriae in kittiwake Rissa tridactyla colonies in relation to the age of the colony and the mechanism of infecting new colonies. Ibis 134:134-141

> Davidson R, Simard M, Kutz SJ, Kapel CMO, Hamnes IS, Robertson LJ (2011) Arctic parasitology: Why should we care? Trends Parasitol 27:239-245

de Meeûs T, McCoy KD, Prugnolle F, Chevillon C, Durand P, Hurtrez-Boussès S, Renaud F (2007) Population genetics and molecular epidemiology or how to 'débusquer la bête'. Infect Genet Evol 7:308-332

> de Meeûs T, Guégan JF, Teriokhin AT (2009) MultiTest V.1.2, a program to binomially combine independent tests and performance comparison with other related methods on proportional data. BMC Bioinformatics 10: 443 doi:10.1186/1471-2105-10-443

Dietrich M (2011) Diversité et adaptation des parasites: formation de races d'hôtes chez la tique Ixodes uriae. PhD dissertation, Université Montpellier 2, Montpellier 
Dietrich M, Gómez-Díaz E, McCoy KD (2011) Worldwide distribution and diversity of seabird ticks: implications for the ecology and epidemiology of tick-borne pathogens. Vector Borne Zoonotic Dis 11:453-470

> Dietrich M, Kempf F, Gómez-Díaz E, Kitaysky AS, Hipfner JM, Boulinier T, McCoy KD (2012) Inter-oceanic variation in patterns of host-associated divergence in a seabird ectoparasite. J Biogeogr 39:545-555

> Dranitsina AS, Telegeev GD, Maliuta SS, Bezrukov FV (2006) Genetic diversity in populations of gentoo penguins (Pygoscelis papua). Tsitol Genet 40:57-62

Dugger KM, Ainley DG, Lyver PO, Barton K, Ballard G (2010) Survival differences and the effect of environmental instability on breeding dispersal in an Adélie penguin meta-population. Proc Natl Acad Sci USA 107: $12375-12380$

Emslie SD, McDaniel JD (2002) Adélie penguin diet and climate change during the middle to late Holocene in northern Marguerite Bay, Antarctic Peninsula. Polar Biol 25:222-229

Evanno G, Regnaut S, Goudet J (2005) Detecting the number of clusters of individuals using the software STRUCTURE: a simulation study. Mol Ecol 14:2611-2620

Eveleigh ES, Threlfall W (1975) The biology of Ixodes (Ceratixodes) uriae White, 1852 in Newfoundland. Acarologia 16:621-635

Forcada J, Trathan PN, Reid K, Murphy EJ, Croxall JP (2006) Contrasting population changes in sympatric penguin species in association with climate warming. Glob Change Biol 12:411-423

Fraser WR, Hofmann EE (2003) A predator's perspective on causal links between climate change, physical forcing and ecosystem response. Mar Ecol Prog Ser 265:1-15

Frenot Y, de Oliveira E, Gauthier-Clerc M, Deunff J, Bellido A, Vernon P (2001) Life cycle of the tick Ixodes uriae in penguin colonies: relationships with host breeding activity. Int J Parasitol 31:1040-1047

> Gauthier-Clerc M, Clerquin Y, Handrich Y (1998) Hyperinfestation by ticks Ixodes uriae: a possible cause of death in adult king penguins, a long-lived seabird. Colon Waterbirds 21:229-233

Gómez-Díaz E, Doherty PF Jr, Duneau D, McCoy KD (2010) Cryptic vector divergence masks vector-specific patterns of infection: an example from the marine cycle of Lyme borreliosis. Evol Appl 3:391-401

> Gómez-Díaz E, Boulinier T, Sertour N, Cornet M, Ferquel E, McCoy KD (2011) Genetic structure of marine Borrelia garinii and population admixture with the terrestrial cycle of Lyme borreliosis. Environ Microbiol 13: 2453-2467

Goudet J (1995) FSTAT (vers. 1.2): a computer program to calculate F-statistics. J Hered 86:485-486

> Goudet J, Raymond M, de Meeûs T, Rousset F (1996) Testing differentiation in diploid populations. Genetics 144: 1933-1940

> Hubisz MJ, Falush D, Stephens M, Pritchard JK (2009) Inferring weak population structure with the assistance of sample group information. Mol Ecol Resour 9:1322-1332

Jensen JL, Bohonak AJ, Kelley ST (2005) Isolation by distance, web service. BMC Genet 6:13 doi:10.1186/14712156-6-13

Kempf F, Boulinier T, de Meeûs T, Arnathau C, McCoy KD (2009) Recent evolution of host-associated divergence in the seabird tick Ixodes uriae. Mol Ecol 18:4450-4462

Le Bohec C, Durant JM, Gauthier-Clerc M, Stenseth NC and others (2008) King penguin population threatened by Southern Ocean warming. Proc Natl Acad Sci USA 105: 2493-2497

> Lee RE Jr, Baust JG (1982) Low-temperature adaptation in an Antarctic tick. Cryobiology 19:658-659

Lynch HJ, Naveen R, Fagan WF (2008) Censuses of penguin, blue-eyed shag Phalacrocorax atriceps and southern giant petrel Macronectes giganteus populations on the Antarctic Peninsula, 2001-2007. Mar Ornithol 36: 83-97

Lynch HJ, Fagan WF, Naveen R (2010) Population trends and reproductive success at a frequently visited penguin colony on the western Antarctic Peninsula. Polar Biol 33: 493-503

> Magalhães S, Forbes MR, Skoracka A, Osakabe M, Chevillon C, McCoy KD (2007) Host race formation in the Acari. Exp Appl Acarol 42:225-238

Mangin S, Gauthier-Clerc M, Frenot Y, Gendner JP, Le Maho Y (2003) Ticks Ixodes uriae and the breeding performance of a colonial seabird, king penguin Aptenodytes patagonicus. J Avian Biol 34:30-34

McCoy KD (2009) Host-parasite determinants of parasite population structure: lessons from bats and mites on the importance of time. Mol Ecol 18:3545-3547

> McCoy KD, Tirard C (2000) Isolation and characterisation of microsatellites in the seabird ectoparasite Ixodes uriae. Mol Ecol 9:2213-2214

McCoy KD, Boulinier T, Tirard C, Michalakis Y (2001) Host specificity of a generalist parasite: genetic evidence of sympatric host races in the seabird tick Ixodes uriae. J Evol Biol 14:395-405

McCoy KD, Boulinier T, Tirard C, Michalakis Y (2003) Hostdependent genetic structure of parasite populations: differential dispersal of seabird tick host races. Evolution 57:288-296

McCoy KD, Boulinier T, Tirard C (2005a) Comparative host-parasite population structures: disentangling prospecting and dispersal in the black-legged kittiwake Rissa tridactyla. Mol Ecol 14:2825-2838

McCoy KD, Chapuis E, Tirard C, Boulinier T, Michalakis Y, Le Bohec C, Le Maho Y, Gauthier-Clerc M (2005b) Recurrent evolution of host-specialized races in a globally distributed parasite. Proc R Soc Lond B 272: 2389-2395

> Morbey YE (1996) The abundance and effects of ticks (Ixodes uriae) on nestling Cassin's auklets (Ptychoramphus aleuticus) at Triangle Island, British Columbia. Can J Zool 74:1585-1589

Olsén B, Jaenson TGT, Noppa L, Bunikis J, Bergström S (1993) A Lyme borreliosis cycle in seabirds and Ixodes uriae ticks. Nature 362:340-342

Polito MJ, Lynch HJ, Naveen R, Emslie SD (2011) Stable isotopes reveal regional heterogeneity in the prebreeding distribution and diets of sympatrically breeding Pygoscelis spp. penguins. Mar Ecol Prog Ser 421: 265-277

Pritchard JK, Stephens M, Donnelly P (2000) Inference of population structure using multilocus genotype data. Genetics 155:945-959

Raymond M, Rousset F (1995) GENEPOP(version 1.2): population genetics software for exact tests and ecumenicism. J Hered 86:248-249

Roeder AD, Marshall RK, Mitchelson AJ, Visagathilagar T and others (2001) Gene flow on the ice: genetic differentiation among Adélie penguin colonies around Antarc- 
tica. Mol Ecol 10:1645-1656

Steig EJ, Schneider DP, Rutherford SD, Mann ME, Comiso JC, Shindell DT (2009) Warming of the Antarctic icesheet surface since the 1957 international geophysical year. Nature 457:459-462

Tavares ES, Baker AJ (2008) Single mitochondrial gene barcodes reliably identify sister-species in diverse clades of birds. BMC Evol Biol 8:81

Trivelpiece WZ, Trivelpiece SG, Volkman NJ (1987) Ecological segregation of Adélie, gentoo, and chinstrap penguins at King George Island, Antarctica. Ecology 68: 351-361

Trivelpiece WZ, Hinke JT, Miller AK, Reiss CS, Trivelpiece SG, Watters GM (2011) Variability in krill biomass links harvesting and climate warming to penguin population changes in Antarctica. Proc Natl Acad Sci USA 108: 7625-7628

Van Oosterhout C, Hutchinson WF, Wills DPM, Shipley P

Editorial responsibility: Hans Heinrich Janssen, Oldendorf/Luhe, Germany
(2004) MICRO-CHECKER: software for identifying and correcting genotyping errors in microsatellite data. Mol Ecol Notes 4:535-538

Wardle DA, Bardgett RD, Callaway RM, Van der Putten WH (2011) Terrestrial ecosystem responses to species gains and losses. Science 332:1273-1277

Weir BS, Cockerham CC (1984) Estimating F-statistics for the analysis of population structure. Evolution 38: $1358-1370$

Williams TD (1995) The penguins. Oxford University Press, Oxford

Wilson N (1967) Mesostigmata: Rhinonyssidae, Halarachnidae (nasal mites); Metastigmata: Ixodidae (ticks). In: Gressit JL (ed) Entomology of Antarctica. American Geophysical Union, Washington, DC, p 41-50

Woehler EJ (1993) The distribution and abundance of Antarctic and Subantarctic penguins. Scientific Committee on Antarctic Research, Cambridge

Submitted: November 30, 2011; Accepted: April 10, 2012

Proofs received from author(s): June 11, 2012 